\title{
Systematic Review of Prognostic Factors for Return to Work in Workers with Sub Acute and Chronic Low Back Pain
}

\author{
Ivan A. Steenstra ${ }^{1,2}(1) \cdot$ Claire Munhall $^{1} \cdot$ Emma Irvin $^{1} \cdot$ Nelson Oranye $^{3} \cdot$ \\ Steven Passmore $^{3}$ - Dwayne Van Eerd ${ }^{1}$ - Quenby Mahood ${ }^{1} \cdot$ Sheilah Hogg-Johnson $^{1}$
}

Published online: 19 September 2016

(c) The Author(s) 2016. This article is published with open access at Springerlink.com

\begin{abstract}
Purpose We systematically reviewed the evidence on factors that predict duration of sick leave in workers after 6 weeks low back pain (LBP) related sick leave. We hypothesized that different factors affect the duration of the leave depending on the time away from work. Methods The review occurred in seven phases: (1) developing the central question, (2) conducting the literature search, (3) identifying relevant publications, (4) quality appraisal, (5) data extraction, (6) evidence synthesis, and (7) knowledge translation. We searched for studies that reported episodes of LBP and sick leave that lasted more than 6 weeks. All included studies reported at least one prognostic factor where return to work was the outcome. Results We identified twenty-two relevant publications. The impact of pain, functional status and radiating pain seems to change with duration of work disability. Workers' recovery expectations remain important after 6 weeks. Modified duties are rarely studied in later phases of work disability. Depression/mental health did not appear to be an important factor in later phases. Workplace physical factors remain important. There is insufficient evidence that pain catastrophising and fear avoidance are predictive factors in later phases. There was moderate evidence for age in the later phases. Functional capacity
\end{abstract}

Electronic supplementary material The online version of this article (doi:10.1007/s10926-016-9666-x) contains supplementary material, which is available to authorized users.

$\triangle$ Ivan A. Steenstra

ivan.steenstra@ryerson.ca

1 Institute for Work and Health, Toronto, ON, Canada

2 Ted Rogers School of Management, Ryerson University, 350 Victoria Street, Toronto, ON M5B 2K3, Canada

3 University of Manitoba, Winnipeg, MB, Canada and claim related factors were supported by some evidence. Discusion Physical demands in the workplace are preventing workers from getting back to work in a timely fashion across phases. The psychosocial work environment is understudied in later phases. Overall, we cannot conclude that prognostic factors change over time.

Keywords Disability - Sick leave · Disability evaluation · Review · Systematic · Prognosis

\section{Background}

Delayed return to work (RTW) is associated with high compensation and treatment

costs. In the United States (US) indirect costs of low back pain (LBP) are estimated to be more than US $\$ 50$ billion per year [1], in the United Kingdom (UK) US \$11 billion [2] and in the Netherlands almost US $\$ 5$ billion [3]. Hoy et al. [4] state that LBP causes more global disability than any other condition and that there is an urgent need for further research to better understand LBP across settings.

Frank et al. [5] proposed a model that classifies three stages in the work disability process: Acute (0-6 weeks); Subacute (6-12 weeks); and Chronic (12+ weeks). Over the course of the work disability process some workers return to work, while others remain off work. Time is needed to recover from injury, but over time RTW could become complicated by a number of factors. Factors that might be predictive at an early stage of RTW might differ from those that are important at a later stage.

We previously published systematic reviews on prognostic factors for duration on sick leave due to acute LBP (with duration of $0-6$ weeks) [6, 7]. These reviews showed that there was strong evidence that the following 
factors had an association with the duration of sick leave: recovery expectations, radiating pain (injury severity), pain (self report), disability (self report), workplace physical factors, and provider type. There was also strong evidence that lifestyle and pain catastrophising had no association with duration of sick leave. Moderate evidence was found for modified duties, workplace psychosocial factors, claim-related factors and content of treatment and there was moderate evidence for no association of previous injury and clinical examination. There was insufficient evidence for age, education, language barriers, depression/mental health, fear avoidance beliefs, work relatedness, workplace-organizational factors and the process of treatment.

This study extends the scope of the previous reviews by systematically reviewing the evidence on factors that predict duration of time away from work at the sub acute and the chronic stage of a LBP related episode of time away from work. The first hypothesis was that there are factors related to LBP, to the worker, to the job and to the psychosocial environment that influence duration of an episode of sick leave.

The second hypothesis was that in the sub acute and even more so in the chronic phase, psychosocial issues will likely become more prominent compared to the acute phase.

\section{Methodology}

\section{Classifying Prognostic Factors}

LBP is considered to be a multidimensional problem. A framework proposed by Loisel et al. [8] further elaborates the structure of the International Classification of Functioning, Disability and Health (ICF) [9-11] to also include factors related to the workplace, healthcare and workers' compensation environment. By applying this framework we further distinguished between predictive factors related to the LBP, the worker, the job and workplace, and the psychosocial environment, specifically to health care services and the workers compensation insurer. These theoretical frameworks structured our analyses and aided in clear reporting to stakeholder groups.

\section{Classifying Outcomes}

The concept of time away from work is highly dependent on legislation and locally-used jargon. In North America, "time away from work", "time on disability benefits" or "disability" is used to define time away from work. In Europe, the phrases "sick leave" and "RTW" are used more often, since disability is used to define functional limitations (for instance as measured by the Roland Morris Disability Questionnaire). These differences were recognized in our search strategy [6].

\section{Search Strategy}

We used an updated search strategy from previous reviews [12-14] (see Online Appendix I) in Medline (OVID), EMBASE (OVID) and PsycINFO (OVID) from inception of each database to 2012. The search was constructed in three broad categories: (1) Prognosis terms, (2) Back Pain terms, and (3) Work/Return To Work terms. The terms within each category were combined with an OR Boolean operator and then the three categories were combined with an AND Boolean operator. The search therefore captured references with at least one term in each of the three categories. As each database is unique, the search was customized accordingly to best utilize the controlled vocabularies of each. Search yields were combined and duplicates were removed. We reviewed the search yield for studies on LBP [12], prognosis [15] and work and stratified the results for each phase of work disability. The references list of all relevant articles and recently published systematic reviews were screened for additional publications. An in-depth comparison of search strategies [15, 16] has shown that our search strategy was broad enough to capture as much relevant literature as possible.

\section{Selection of Studies}

Two reviewers independently selected studies that met similar inclusion and exclusion criteria as in our previous reviews $[6,17]$, except for the disability phase:

1. Studies that included subjects with an episode of LBP and sick leave, with duration of more than 6 weeks at inclusion of cohort;

2. Studied the relation between at least one prognostic factor and outcome; and

3. Measured outcomes in absolute terms (rate), relative terms (odds ratio, rate ratio, hazard ratio), survival curve or duration of sick leave.

First, titles and abstracts were screened, followed by possibly relevant full articles. A third reviewer resolved disagreements if consensus between two reviewers could not be reached.

The third reviewer (IS or SHJ) used his/her knowledge and experience in the field of prognosis research to weigh the different view of assessing studies for suitability. The initial reviewers reached consensus in most cases and the third reviewer only had to be consulted in a minimal number of cases where reviewers erred on the side of caution. 


\section{Quality Assessment}

Two reviewers independently scored the quality of included studies. The quality was appraised using a tool developed in our previous reviews [7] (see Online Appendix II). Item 24 asked the reviewer for a general appraisal of study quality using a 11 point VAS scale. This item was not used in the assessment of study quality, because of a lack of agreement with the overall scale score. The third reviewer (IS or SHJ) used his/her knowledge and experience in the field of prognosis research to weigh the different view of assessing studies for suitability. The initial reviewers reached consensus in most cases and the third reviewer only had to be consulted in a minimal number of cases where reviewers erred on the side of caution.

\section{Data Extraction}

Due to heterogeneity in studies we did not conduct a metaanalysis. The evidence for each prognostic factor was therefore presented in a descriptive manner.

The information extracted from each study included definition of prognostic factor and outcome, country, setting, association estimate, sample size. Risk of RTW was recalculated to the risk of no RTW. This means that a ratio larger than 1 means a delay in time until RTW.

\section{Levels of Evidence}

Relevant studies were grouped by prognostic factors and the level of evidence for each prognostic factor was determined by into consideration the quality ratings of each study and the consistency of findings across studies in terms of significance and direction of association across the different studies examining each particular prognostic factor. The criteria for describing the level of evidence for each prognostic factor is based on van Hoogendoorn et al. [18] rating system and is consistent with our previous reviews on prognosis in RTW in the acute phase of LBP $[6,19]$ :

- Strong evidence consistent findings in multiple high quality studies.

- Moderate evidence consistent findings in one high quality study and one or more lower quality studies, or in multiple lower quality studies.

- Insufficient evidence only one study available or inconsistent findings in multiple studies.

\section{Results}

The initial search yielded 5027 research papers, after duplicates were removed. After the screening of all titles and abstracts, 939 papers were retrieved for a more full text review. Seventy-eight publications met all of the inclusion criteria. Sixteen publications were from the chronic phase, six were from the sub acute phase, 37 were from the acute phase and 19 studies were either in populations from different phases or did not report the duration of sick leave.

Publications that included cases from the sub acute phase were from Canada (4), the USA (1) and Norway (1). Publications that included cases from the chronic phase were from Canada (3), the USA (6), Norway (1), Netherlands (3) and from an international study in Denmark, Germany, Israel, Sweden, the Netherlands, and the United States (3). (See Fig. 1.)

In the sub acute phase the mean quality score was: 15.5 (range 14-19). In the chronic phase the mean quality score was: 14.8 (range 9-21). Five of these studies were high quality (QA score $>16$ ), 13 were of moderate quality (QA score between 13 and 16), and seven were of lower quality $(\mathrm{QA}<13)$.

481 prognostic factors were considered were studied across all phases. Prognostic factors were grouped in a number of team meetings into clinical, personal psychosocial, work related psychosocial, and claim related prognostic factors. Within each category, factors or tools measuring the same or very similar constructs (for instance different methods to report on physical demands) were merged resulting in 43 different constructs that we report on. See Table 1 for detailed characteristics of the included studies.

\section{Evidence on Prognostic Factors}

We present the results on the evidence for association for factors in the chronic phase followed by those from the subacute phase according to the categories we determined.

A summary of the evidence on prognostic factors is presented in Table 2.

\section{Results on Clinical Prognostic Factors}

Sex and Age

Chronic phase There is moderate evidence of negative association between male sex and RTW from 1 medium quality [20] and one high quality study [21]. Notably, one high quality study [22] and one low quality study [23] did not find an association between sex and RTW. However, since they did not find an association between female sex and RTW, this is not a contradictory finding and could be the result of small sample sizes.

Sub acute phase There is strong evidence for no association between sex and RTW in the sub acute phase based on two high quality studies [24, 25] and a medium quality study [26]. Only one medium quality study [27] found a negative association with male sex. 
Fig. 1 Flowchart chronicling the search process

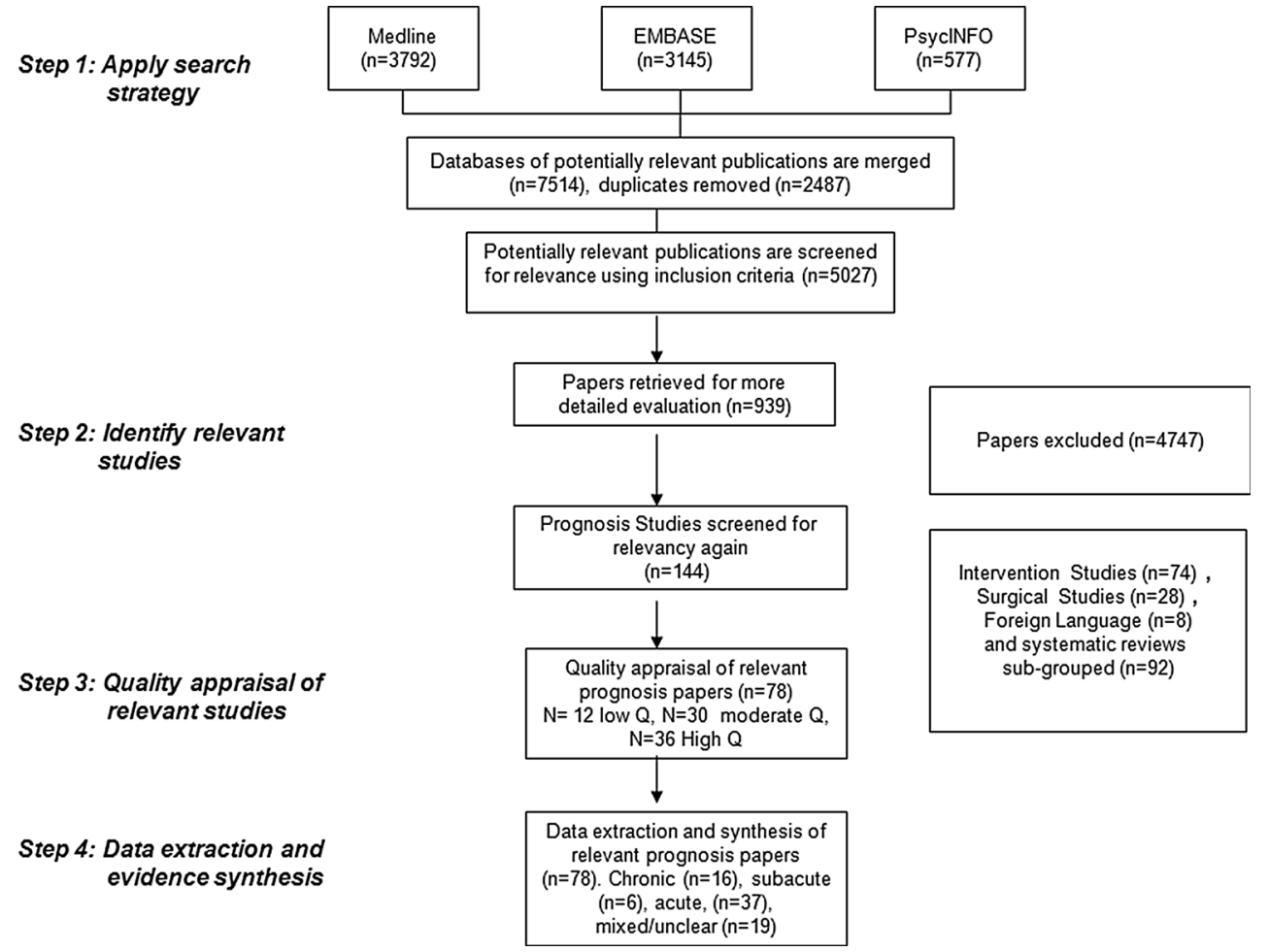

Chronic phase There is moderate evidence for a negative association between older age and RTW from a high quality study [24] and a medium quality study [26].

Most studies reported a negative association between older age and RTW, although not all were statistically significant [21-23].

Subacute phase There is moderate evidence for older age from one high quality study [28], two medium quality publications [20,29] and one lower quality publication [30] that reported a negative association between age and RTW.

\section{Pain and Function}

Chronic phase Radiating pain was not studied in the selected studies. There is moderate evidence that pain intensity has a negative association with RTW in the chronic phase from one high quality study [21], one moderate quality study [29-31] and two lower quality studies [23, 32]. One lower quality study did not report on pain [33]. There is moderate evidence for a negative association between function and RTW from one lower quality [23] and two medium quality studies [20, 34]. With respect to the results of functional capacity evaluation, there is moderate evidence for a positive association with RTW, based on one high quality study (cardiovascular fitness) [35] and one medium quality study (trunk flexibility) [20], however two lower quality studies $[23,32]$ found no significant association with RTW.

Sub acute phase There is moderate evidence for no association of radiating pain with RTW [25]. There is strong evidence for no association with function from two high quality studies [24, 25] and two medium quality studies $[36,37]$. There is strong evidence for no association between pain and RTW as well from two high quality studies $[24,25]$ and one medium quality study [36]. There is moderate evidence for a positive association of a higher score on a functional capacity evaluation (FCE) and RTW from one publication reporting on two cohorts of injured workers [37]. There was one study that observed a negative association between pain observation and RTW [25].

\section{Treatment Related Clinical Factors}

Chronic phase There is strong evidence from 4 high quality studies [21, 22, 28, 38] and one lower quality study [23] that a delay in referral to intervention was associated with a delay in RTW. One high quality study [22] found a positive association of insurer mandated rehabilitation and one medium quality study [31] found a positive association of several medical interventions (surgery between 4 and 12 months, pain medication and exercise therapy), which results in moderate evidence for a positive association between "intervention" and RTW.

Sub acute phase There is moderate evidence for a positive association between intervention and RTW from one high quality (prior physiotherapy) [24] and two medium quality studies (time in work hardening program and stretching) [26, 27]. 


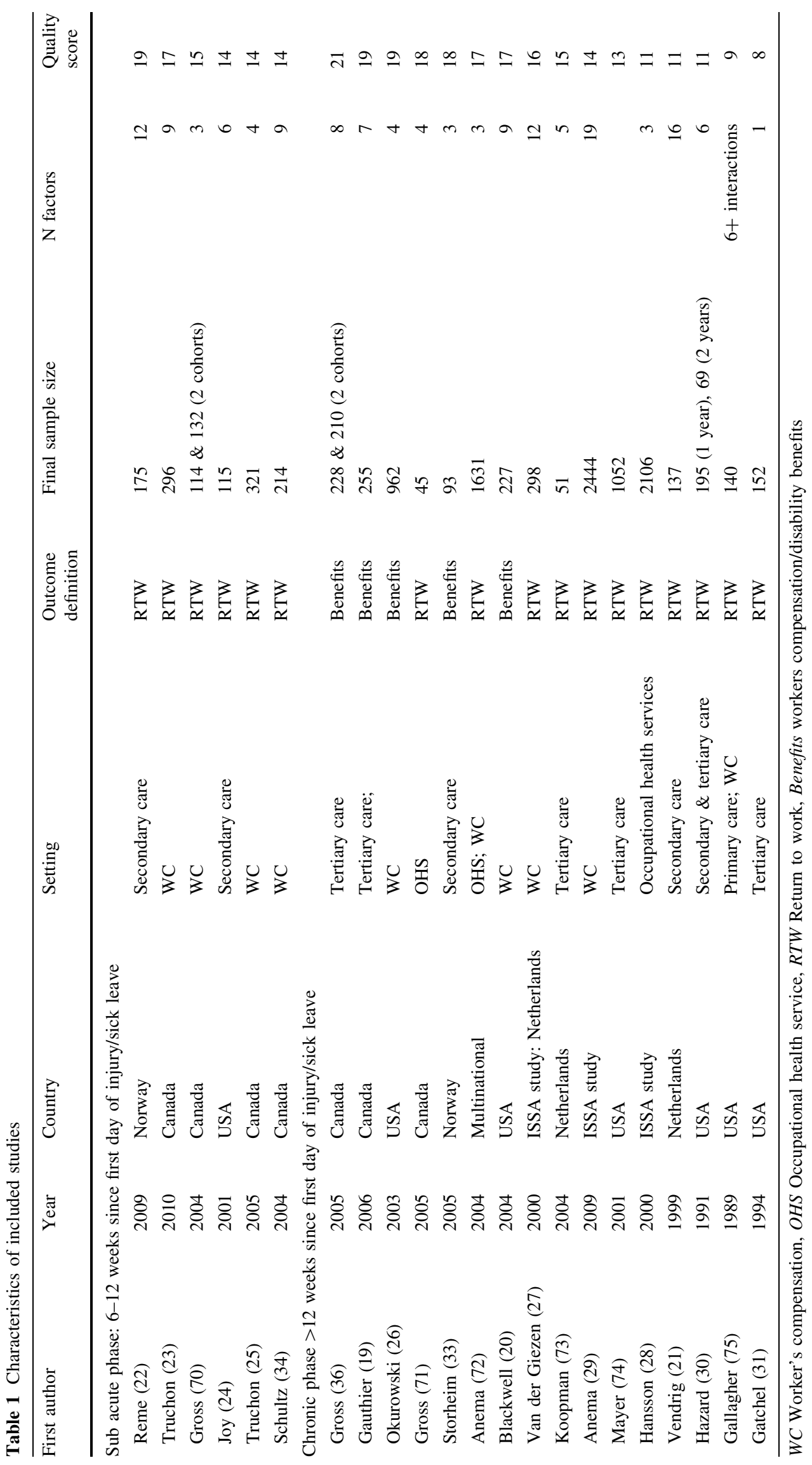


Table 2 Prognostic factor evidence table

\begin{tabular}{|c|c|c|c|}
\hline Prognostic factor & Phase & Evidence & Level of evidence \\
\hline \multicolumn{4}{|l|}{ Clinical prognostic factors } \\
\hline \multirow[t]{2}{*}{ Sex } & Chronic & $2 \mathrm{H}, 1 \mathrm{M}, 1 \mathrm{~L}$ & $\begin{array}{l}\text { Moderate for negative association with male } \\
\text { sex }\end{array}$ \\
\hline & Subacute & $2 \mathrm{H}, 1 \mathrm{M}, 1 \mathrm{M}$ & Strong for no association \\
\hline \multirow[t]{2}{*}{ Age } & Chronic & $2 \mathrm{H}, 1 \mathrm{H}, 2 \mathrm{M}, 2 \mathrm{~L}, 1 \mathrm{~L}$ & Moderate for negative association \\
\hline & Subacute & $1 \mathrm{H}, 1 \mathrm{M}$ & Moderate for negative association \\
\hline Comorbidity & Chronic & $1 \mathrm{M}$ & Insufficient \\
\hline Diagnosis & Chronic & $1 \mathrm{~L}$ & Insufficient \\
\hline Radiating & Subacute & $1 \mathrm{H}$ & Moderate for no association \\
\hline \multirow[t]{2}{*}{ Pain Intensity } & Chronic & $1 \mathrm{H}, 2 \mathrm{M}, 2 \mathrm{~L}$ & Moderate for negative association \\
\hline & Subacute & $2 \mathrm{H}, 1 \mathrm{M}$ & Strong for no association \\
\hline \multirow[t]{2}{*}{ Functional status } & Chronic & $2 \mathrm{M}, 1 \mathrm{~L}$ & Moderate for negative association \\
\hline & Subacute & $2 \mathrm{H}, 2 \mathrm{M}$ & Strong for no association \\
\hline \multirow[t]{2}{*}{ Functional status-FCE } & Chronic & $1 \mathrm{H}, 1 \mathrm{M}, 2 \mathrm{~L}$ & Moderate for positive association \\
\hline & Subacute & $\begin{array}{l}2 \mathrm{M}(2 \text { cohorts, one } \\
\text { publication) }\end{array}$ & Moderate for positive association \\
\hline Pain observation & Subacute & $1 \mathrm{M}$ & Insufficient \\
\hline Delay in referral & Chronic & $4 \mathrm{H}, 1 \mathrm{~L}$ & Strong for negative association \\
\hline \multirow[t]{2}{*}{ Intervention } & Chronic & $1 \mathrm{M}, 1 \mathrm{H}$ & Moderate for positive association \\
\hline & Subacute & $1 \mathrm{H}, 2 \mathrm{M}$ & Moderate for positive association \\
\hline \multirow[t]{2}{*}{ Health } & Chronic & $2 \mathrm{M}, 1 \mathrm{~L}$ & Moderate for positive association \\
\hline & Subacute & $1 \mathrm{M}$ & Insufficient \\
\hline Lifestyle & Chronic & $1 \mathrm{~L}$ & Insufficient \\
\hline \multicolumn{4}{|l|}{ Psychosocial prognostic factors } \\
\hline \multirow[t]{2}{*}{ Expectation of RTW } & Chronic & $2 \mathrm{M}, 1 \mathrm{~L}$ & Insufficient \\
\hline & Subacute & $1 \mathrm{H}, 1 \mathrm{M}$ & Moderate for positive association \\
\hline \multirow{2}{*}{$\begin{array}{l}\text { Fear avoidance/Pain catastrophising/ } \\
\text { Cognitive appraisal/Coping }\end{array}$} & Chronic & $1 \mathrm{H}, 1 \mathrm{M}, 1 \mathrm{~L}$ & Moderate evidence for negative association* \\
\hline & Subacute & $1 \mathrm{H}, 1 \mathrm{M}$ & Moderate evidence for negative association* \\
\hline Distress & Subacute & $1 \mathrm{H}$ & Insufficient \\
\hline Depression & Chronic & $1 \mathrm{H}, 1 \mathrm{~L}$ & Moderate for no association \\
\hline Mental Health & Chronic & $2 \mathrm{~L}, 2 \mathrm{~L}$ & Insufficient \\
\hline \multicolumn{4}{|l|}{ Social workplace prognostic factors } \\
\hline \multirow[t]{2}{*}{ SES } & Chronic & $2 \mathrm{H}, 1 \mathrm{M}, 1 \mathrm{~L}$ & Strong evidence for positive association \\
\hline & Subacute & $1 \mathrm{H}$ & Insufficient \\
\hline \multirow[t]{2}{*}{ Physical demands } & Chronic & $1 \mathrm{H}, 1 \mathrm{M}$ & Moderate evidence for positive association \\
\hline & Subacute & $1 \mathrm{H}$ & Insufficient \\
\hline Modified duties & Chronic & $1 \mathrm{M}$ & Insufficient \\
\hline \multirow[t]{2}{*}{ Social support } & Chronic & $1 \mathrm{H}$ & Insufficient \\
\hline & Subacute & $1 \mathrm{M}$ & Insufficient \\
\hline Job satisfaction & Chronic & $1 \mathrm{M}$ & Insufficient \\
\hline Attorney involvement & Chronic & $2 \mathrm{H}$ & Strong evidence for negative association \\
\hline Worker's compensation & Chronic & $2 \mathrm{H}, 1 \mathrm{M}, 1 \mathrm{~L}$ & Strong evidence for negative association \\
\hline
\end{tabular}

$H$ High quality, $M$ Moderate quality, $L$ Low quality, SES Socio economic status, *Heterogeneity in measures

\section{Health Related Clinical Factors}

Chronic phase There is moderate evidence for health measures on RTW from two medium quality (general health and physical function) [29, 35] and one lower quality (social function in Sweden and USA) [30] studies.

Subacute phase There was insufficient evidence for a positive association of health on RTW because only one 
study [36] found a positive association between this factor and RTW in this phase.

Chronic phase A medium quality study [32] found no association between lifestyle (smoking) and RTW.

\section{Results on Personal Psychosocial Factors}

\section{Recovery Expectations}

Chronic phase Researchers from one international study [29-31] reported inconsistently on the one item question from the work ability index [39] that asks about expectations of RTW. We conclude that there is insufficient evidence for recovery expectations in the chronic phase.

Sub acute phase One high quality study [24] found a positive association of expectations of RTW with RTW, even though only the 3 months mark was statistically significant. Another medium quality study [36] also found a negative association of low expectations with RTW. We consider this as moderate evidence for the association between recovery expectations and RTW.

\section{Pain Catastrophising, Fear Avoidance, Coping}

Chronic phase One medium quality study [35] found a negative association between FAB-Q and time on benefits. The high quality study from Gauthier et al. [21] found no association of fear of movement on time on benefits. One high quality study [21] reported a negative association between pain catastrophising and time on benefits. Another medium quality study [20] found a negative association between coping and RTW. We argue there is moderate evidence for a negative association for the concept of fear of movement, since different, but conceptually similar, measures were used in a limited number of studies.

Sub acute phase Again, different, but conceptually similar, measures were used in a limited number of studies, resulting in limited evidence for all these factors. One high quality study [25] found a rather strong association of the score on the Fear Avoidance Beliefs Questionnaire (FABQ) and RTW (odds ratios of 5 and 3). This study [25] also found a negative association of cognitive appraisal on RTW. One medium quality publication from the same first author [27] found a negative association between pain catastrophising and RTW. Again, there is moderate evidence for a negative effect of the fear of movement concept.

\section{Distress, Depression, Mental Health}

Sub acute phase Only distress was examined in this phase in one high quality study [25], which reported a negative association with RTW. There is insufficient evidence for the association between distress and RTW in the sub acute phase because only one study considered this category of factors.

Chronic phase One high quality [21] and one lower quality [23] studies found no statistically significant association between depressive symptoms and RTW, resulting in moderate evidence for no association for this factor. The high quality study examined 7 constructs in a population with 98 events, which indicates sufficient power for this study [40].

Chronic phase Three low quality studies [23, 32, 33] examined the association between mental health and RTW. One of the studies [23] reported a negative association, while the other two found no effect of mental health on RTW. Therefore, there is insufficient evidence for a negative association between mental health and the outcome.

\section{Results on Work Related Psycho-Social Factors}

\section{Socioeconomic Status, Physical Demands, and Modified Duties}

These factors are reported in the same section because they are related. Workers that are classified as having lower socio economic status (SES), often have more physically demanding jobs. Modified duties are often used to (temporarily) mitigate the negative associations of physically demanding work. Unfortunately, none of the studies in the review measured these factors simultaneously.

Sub acute phase One high quality study [24] found a positive association between lower physical demands and RTW. The same study found no association between education and RTW. In summary there is insufficient evidence for an association with RTW in the sub acute phase, due to a limited number of high quality studies.

Chronic phase One high quality [22], one lower quality [30] and one medium quality [31] publications (all from the ISSA study) showed a positive association between lower physical demands and faster RTW. Due to the limited number of high quality studies, there is moderate evidence for physical demands on RTW in the chronic phase. We found strong evidence for SES, although it was measured in rather different ways in different studies. One high quality study [28] reported a negative association between language barriers and RTW. Another high quality publication [22] found a positive association of higher education with RTW. A medium quality publication [29] found a positive association between being a breadwinner and RTW. One lower quality publication [23] only reported a non significant association for education. One medium quality publication [31] reported positive associations between modified duties and RTW. However, due to the limited number of studies there is insufficient evidence for 
the association between modified duties and RTW in the chronic phase.

\section{Social Support, Skill Discretion, Job Satisfaction}

Sub acute phase one medium quality publication [36] reported no association between social (co-worker) support and RTW. That same study reported a negative association between skill discretion and RTW.

Chronic phase One high quality study [22] reported a non significant (positive) association of being married on RTW, but also reported a not statistically significant association between skill level and RTW. A medium quality publication [29] reported a positive association between job satisfaction and RTW, oddly the other publications from this study $[30,31]$ did not report on it.

In summary, there is insufficient evidence due to the lack of high quality studies for all of the work related psychosocial factors in both the sub acute and chronic phases.

\section{Claim Related Factors}

Chronic phase Two high quality studies reported a negative association between attorney involvement and RTW $[22,28]$, which results in strong evidence for the association between attorney involvement and RTW. Anema et al. [31] in their cross jurisdictional (medium quality) study, found that workers compensation policies and practice are associated with RTW outcomes. However, since only one study looked into policies and practices, this factor is supported by insufficient evidence.

Sub acute phase One medium quality study [36] in the sub acute phase found a negative association between Workers Compensation Board and employer response and RTW. This results in insufficient evidence to support this factor due to a lack of high quality studies.

\section{Discussion}

Our first hypothesis was that there are factors related to LBP, to the worker, to the job and to the psychosocial environment that influence duration of an episode of sick leave. The results presented in Table 2 show that factors within the clinical, psychosocial and workplace categories are associated with RTW. Understanding these factors can help practitioners dealing with patients during the RTW process. There does not seem to be consensus between researchers on a core set of prognostic factors that should be included in prognostic studies in LBP and work disability in particular. While some may argue for the use of meta-analysis, like we did in our first review [6], we deemed meta-analysis inappropriate for this review because of the lack of consensus on adjustment of confounders. Moreover, studies measured factors in different ways, there was inconsistency in reporting methods, and a large variability in quality of the studies.

Our second hypothesis was that in the sub acute and even more so in the chronic phase, psychological and social issues would likely become more prominent compared to the acute phase. This hypothesis cannot be confirmed, mainly due to the lack of high quality studies and a lack of consensus among researchers on what to measure, how to measure, and how to analyze the associations. In our previous reviews [6, 17] we found strong evidence for no association of 'pain catastrophising scale' with RTW. We hypothesized that pain catastrophising might play a role at a later stage in the work disability process. However, there are not enough high quality studies to go beyond moderate evidence for any of the pain catastrophising and fear avoidance factors in later phases.

\section{Limitations of the Literature}

The psychosocial work environment is clearly understudied in later phases. There has been lack of consensus among researchers on how to measure psychosocial constructs and how to analyze the data based on the available theoretical models [41]. Considering the theoretical underpinnings of the fear avoidance model (FAB) [41], straightforward predictive analysis might not be appropriate and techniques that take the complexity of concepts and their interrelationships in the FAB model into account might be preferred.

In the acute phase, we found strong evidence for an association between radiating pain-distinctly different from 'non-specific' low-back pain-and RTW [17]. Surprisingly, this factor was only examined in one study [25] from the sub acute phase and in none from the chronic phase. More research seems warranted based on the importance of this factor in the acute phase.

Unlike in our previous review [17], workplace factors were often not considered in the later phases. The related factors: SES, physical demands and modified duties were examined in a number of studies and, despite the crudeness of measures the results show some association with RTW.

For treatment related factors and for the effect found for modified duties, it should be noted that a prognostic study is not the most appropriate study design to examine effectiveness of interventions. Results on effectiveness of interventions can be biased in many ways when an appropriate control group is missing. The association of a delay of referral could very well be caused by immortal time bias [42] or time dependent bias [43, 44] since none of the studies applied time dependent analysis to examine this 
bias. Those that receive intervention are likely to differ from those who do not receive intervention either at baseline or over time.

\section{Strengths and Limitations of this Review}

The strength of this systematic review is that we restricted the analysis to studies with a defined inception point. In an inception cohort, patients are included in the study at the same point in the course of their disease. In many studies on RTW the study population consists of a mixture of workers on sick leave and workers still at work at the time of inception. The number of patients at work during followup depends on both this mixture and on the presence of prognostic factors. Making inferences about the prognosis of RTW from such mixed studies may be misleading. It might be, however, that some researchers do not agree with the phases of disability [45] we used, as a framework for analysis in this review. The cut-offs of 6 and 12 weeks from the Frank et al. publication [45] are somewhat data driven: based on the median and $75^{\text {th }}$ percentile. Populations in different settings and jurisdictions have shown to have different medians and $75^{\text {th }}$ percentiles $[46,47]$ which could have important consequences for the effectiveness of interventions [46]. This classification of time on work related benefits has been extrapolated to outcomes of return to work and functional disability which might not always be appropriate [48].

The seminal paper by Frank et al. [45] seems to assume the outcome "end of benefits" to have a clear inception and a firm endpoint, more recent studies on recurrences [49] and trajectories [50] of low back pain have argued successfully that this is not always the case."

For this review we used the quality assessment that we used in our previous reviews on the acute phase $[6,17]$ to have a consistency in methods. Based on our experience, we recognise that further research is needed in the development of a tool to assess the quality of prognostic research [51].

A prognostic study is not the most appropriate study design to examine the effectiveness of interventions. Especially because immortal time bias [42] or time dependent bias $[43,44]$ are not considered by the selected studies, and because those that receive intervention are likely to differ from those who do not receive intervention either at baseline or over time. Our findings on interventions should be interpreted with that limitation in mind.

Due to the time passed because of the magnitude of the review and the complicated analysis an update of the literature would be worthwhile, however we had to postpone publication because of knowledge transfer workshops and the development of a handbook for our funder. A quick screen of an updated search revealed few new high quality studies that could impact our findings in a substantial way. Some new findings on the importance opioid use in workers compensation settings are in our previous study [19], it should be noted that most of these studies were in the acute phase.

\section{Comparison of Factors in Different Phases}

Workers' recovery expectations seem important in later phases of work disability, despite a lack of high quality studies. It makes sense to ask an injured worker about their expectation for RTW. Unfortunately, there is no consensus among researchers on how to do so, nor have any of the questions used in the studies undergone psychometric testing. However, predictive validity was confirmed in all studies.

The impact of pain, functional status and radiating pain changes with duration when compared to the results from our review on the acute phase [17]. This is somewhat puzzling, although it could be that after some time, when the worst pain has subsided, other factors become more prominent. Workplace physical factors remain important over the entire course of work disability. Therefore, an injured worker should always be asked about the work he/ she did when he/she hurt his/her back and/or what kind of job he/she will return to.

The factors 'self report of disability' $[47,52-60]$ and 'pain intensity' [36, 52-65] were supported by strong evidence in the acute phase, but the evidence is less clear in the sub acute phase [24, 25, 36, 37]. In the chronic phase, there is moderate evidence for a negative association of functional status [20, 23, 34] and of pain Intensity [21, 23, 29-33]. This might indicate a somewhat puzzling U-shape relationship between these factors and RTW over time. It could also be explained by the fact that studies adjust for different confounders.

One factor that was supported by strong evidence in the acute phase is the treatment-related factor: content of care $[47,52,53]$. In other words, it matters with which healthcare provider the worker is in contact. We found moderate evidence for an association between treatment and RTW in the sub acute phase [24, 26, 27]. A delay in referral to intervention was associated with a delay in RTW [21-23, 28, 38]. Overall, experience with and content of treatment matters $[22,31]$ across all phases.

One prognostic factor that was not considered in the acute phase was the impact of functional capacity evaluations on RTW. In the sub acute phase, moderate evidence was found for an association with RTW [20, 23, 32, 35]. In the chronic phase we found moderate evidence for a positive association of a higher score on a functional capacity evaluation (FCE) on RTW [37]. It is not clear whether a full assessment of functional capacity is needed and 
whether it also predicts sustained RTW [66-68]. It should be noted that not only functional capacity evaluation systems were used in work disability assessments, but more traditional "objective measures" of functional capacity like a sub maximal bike ergometer test [35] and trunk flexibility [20] were also included.

In the acute phase, we found moderate evidence for no association of depression on RTW [36, 54, 58]. In the sub acute phase, a negative association between distress and RTW was reported [25]. In the chronic phase, one high quality [21] and one lower quality study [23] found no statistically significant association between depressive symptoms and RTW, resulting in moderate evidence for no association of depressive symptoms on RTW. These findings are consistent with the findings from the acute phase, although there are only a limited number of studies available. Some injured workers might suffer from mental health issues, but scores on different questionnaires do not seem to predict RTW.

Earlier [6, 17], we found that the offer of modified duties, or workplace accommodation improved RTW outcomes $[52,58,69]$. The evidence is not as strong in later phases, mainly because the factor does not seem to be considered by many researchers [31]. Also, when considering the evidence from the intervention literature [70], modified duties should be considered for RTW of injured workers. Timing of the intervention seems best in the acute phase $[6,17]$, although it might also be effective in the late phases [71].

Physical demands are often measured by occupation in the acute phase [52, 54]. Those classified as having more physical jobs are slower to return to work where self-reported physical demands were not associated with RTW [17]. In the later phases, very few studies examined the factor physical demands resulting in insufficient evidence in the sub acute phase [24] and moderate evidence in the chronic phase [22, 30, 31]. We did find strong evidence for SES on RTW [22, 23, 28, 29]. If SES is considered a proxy for physical demands at work, the association between physical demands and RTW seems consistent across phases and should be taken into consideration in the RTW process. Future research on RTW in the later phases of work disability should examine physical demands by using more objective measures.

Job satisfaction was supported by strong evidence in our previous review [17]. It was not examined in the sub acute phase and only one publication [29] reported on it in the chronic phase. The impact of job satisfaction might diminish after a longer time away from the job; however evidence for that hypothesis is lacking.

We found insufficient evidence for an association between age and sex and RTW in acute LBP [17]. There is moderate evidence for a negative association of older age on $\operatorname{RTW}[24,26]$ in the sub acute phase. In the chronic phase, most studies also reported a negative association. Across all phases, the evidence is conflicting and calendar age might not be the most appropriate measure to capture the concept.

In the sub acute phase we found strong evidence for no association of sex on RTW [24-27]. However, one medium quality study found a longer time until RTW in men [26]. There is moderate evidence for an association between male sex and RTW [20, 21] in the chronic phase. Although two studies [22, 23] did not find an association between sex and RTW, this is not contradictory and could be due to small sample sizes. Overall, the association between sex and RTW is inconsistent across phases and might be the result of gender specific workplace based exposures [72].

\section{Future Research}

Prognostic research in work disability prevention would benefit from consensus among research and practitioners on what factors are deemed important and how they should be measured and analysed. Claim-related factors are supported by strong evidence in the chronic phase, and in all cases, are related with delays and experiences in the claims process. This factor was not considered in earlier phases [17]. Some of the claim related factors might be time dependent: they start to play a role at later stages of work disability due to negative side effects of being in the administrative and adjudicative process that happens alongside the RTW process. Further study into claim-factors seems justified.

When presenting the findings from our review to practitioners, it was clear that there is little consensus on what "psychosocial" means in research but great consensus on the importance of the construct in practise. There seems to be a clear disconnect between research and practice that should be resolved.

Understanding of the importance of different prognostic factors at various times in the RTW process can inform stakeholders about the most appropriate actions that can be taken to improve RTW outcomes. To transfer the messages from this review we have presented the findings in a number of workshops. Based on the feedback from stakeholders we are currently developing a Handbook on Prognosis of RTW in LBP for use in practise. The handbook emphasizes the role of recovery expectations and the importance of the workplace and physical demands on the job, and provides suggestions to uncover these constructs when dealing with injured workers trying to RTW. The impact of providing such information to work disability practitioners should be studied. 
Acknowledgments Supported by a Grant from the Workplace Research and Innovation Program of the Workers Compensation Board of Manitoba. We would like to thank Agatha Chandran, Bruce Cielen and colleagues for their help in identifying stakeholders in Manitoba, and all the stakeholders that attended our workshop and the Department of Occupational Therapy, University of Manitoba for hosting the workshop.

\section{Compliance with Ethical Standards}

Conflict of interest This review was funded through a grant from Workplace Research and Innovation Program of the Workers Compensation Board of Manitoba. Ivan Steenstra, Claire Munhall, Nelson Oranye, Dwayne Van Eerd, Quenby Mahood, Emma Irvin and Sheilah Hogg-Johnson have no conflicts of interest to declare. Steven Passmore held the Canadian Chiropractic Research Foundation Professorship in Spine Biomechanics and Human Neurophysiology, in the College of Rehabilitation at the University of Manitoba from the Canadian Chiropractic Research Foundation. As of 2013, the Institute for Work and Health operates with core funding from the Province of Ontario. The stewardship of this funding lies with the Ontario Ministry of Labour (MOL). Previously, the Institute operated with core funding support from the Workplace Safety and Insurance Board (WSIB).

Open Access This article is distributed under the terms of the Creative Commons Attribution 4.0 International License (http://crea tivecommons.org/licenses/by/4.0/), which permits unrestricted use, distribution, and reproduction in any medium, provided you give appropriate credit to the original author(s) and the source, provide a link to the Creative Commons license, and indicate if changes were made.

\section{References}

1. Frymoyer JW, Cats-Baril WL. An overview of the incidences and costs of low back pain. Orthop Clin North Am. 1991;22:263-71.

2. Maniadakis N, Gray A. The economic burden of back pain in the UK. Pain. 2000;84:95-103.

3. van Tulder MW, Koes BW, Bouter LM. A cost-of-illness study of back pain in The Netherlands. Pain. 1995;62:233-40.

4. Hoy D, March L, Brooks P, Blyth F, Woolf A, Bain C, et al. The global burden of low back pain: estimates from the Global Burden of Disease 2010 study. Ann Rheum Dis. 2014;73:968-74.

5. Frank JW, Brooker AS, DeMaio SE, Kerr MS, Maetzel A, Shannon HS, et al. Disability resulting from occupational low back pain Part II: What do we know about secondary prevention? A review of the scientific evidence on prevention after disability begins. Spine. 1996;21:2918-29.

6. Steenstra IA, Verbeek JH, Heymans MW, Bongers PM. Prognostic factors for duration of sick leave in patients sick listed with acute low back pain: a systematic review of the literature. Occup Environ Med. 2005;62:851-60.

7. Steenstra IA, Irvin E, Heymans MW, Mahood Q, Hogg-Johnson S. Systematic review of prognostic factors for workers' time away from work due to acute low-back pain: an update of a systematic review. Final report to WCB Manitoba. 2011. https:// www.iwh.on.ca/system/files/sys-reviews/sys_review_acute_lbp_ 2011b.pdf.

8. Loisel P, Durand MJ, Berthelette D, Vezina N, Baril R, Gagnon D, et al. Disability prevention-New paradigm for the management of occupational back pain. Pract Dis Manag: Dis Manag Health Outcomes. 2001;9:351-60.
9. Wasiak R, Young AE, Dunn KM, Cote P, Gross DP, Heymans $\mathrm{MW}$, et al. Back pain recurrence: an evaluation of existing indicators and direction for future research. Spine. 2009;20(34):970-7.

10. Young AE, Roessler RT, Wasiak R, McPherson KM, van Poppel MNM, Anema JR. A developmental conceptualization of return to work. J Occup Rehab. 2005;15:557-68.

11. World Health Organization. The international classification of functioninjg, disability and health, ICF. Geneva: World Health Organ; 2001.

12. Furlan AD, Pennick V, Bombardier C, Van TM. 2009 updated method guidelines for systematic reviews in the Cochrane Back Review Group. Spine (Phila Pa 1976). 2009;34:1929-41.

13. Haynes RB, Wilczynski N, McKibbon KA, Walker CJ, Sinclair JC. Developing optimal search strategies for detecting clinically sound studies in MEDLINE. J Am Med Inform Assoc. 1994;1:447-58.

14. McKibbon KA, Walker-Dilks C, Haynes RB, Wilczynski N. Beyond ACP Journal Club: how to harness MEDLINE for prognosis problems. ACP J Club. 1995;123:12-4.

15. Wilczynski NL, Haynes RB. Developing optimal search strategies for detecting clinically sound prognostic studies in MEDLINE: an analytic survey. BMC Med. 2004;9(2):23.

16. Wong SS, Wilczynski NL, Haynes RB, Ramkissoonsingh R, Hedges Team. Developing optimal search strategies for detecting sound clinical prediction studies in MEDLINE. AMIA Annu Symp Proc. 2003;728-32.

17. Steenstra IA, Busse JW, Hogg-Johnson S. Predicting return to work for workers with low-back pain. In: Loisel P, Anema JR, Pransky GS, editors. Work disability prevention handbook. New York: Springer; 2013.

18. Hoogendoorn WE, van Poppel MN, Bongers PM, Koes BW, Bouter LM. Systematic review of psychosocial factors at work and private life as risk factors for back pain. Spine. 2000; 15(25):2114-25.

19. Steenstra IA, Busse JW, Tolusso D, Davilmar A, Lee H, Furlan $\mathrm{AD}$, et al. Predicting time on prolonged benefits for injured workers with acute back pain. J Occup Rehabil. 2015;25(2): 267-78.

20. Koopman FS, Edelaar M, Slikker R, Reynders K, van der Woude LH, Hoozemans MJ. Effectiveness of a multidisciplinary occupational training program for chronic low back pain: a prospective cohort study. Am J Phys Med Rehabil. 2004;83:94-103.

21. Gauthier N, Sullivan MJ, Adams H, Stanish WD, Thibault P. Investigating risk factors for chronicity: the importance of distinguishing between return-to-work status and self-report measures of disability. J Occup Environ Med. 2006;48:312-8.

22. Blackwell TL, Leierer SJ, Haupt SS, Kampitsis A, Wolfson JR. Prediction of vocational outcomes for workers' compensation claimants with back injury. J Appl Rehabil Couns. 2004;35(2):32-8.

23. Vendrig AA. Prognostic factors and treatment-related changes associated with return to work in the multimodal treatment of chronic back pain. J Behav Med. 1999;22:217-32.

24. Reme SE, Hagen EM, Eriksen HR. Expectations, perceptions, and physiotherapy predict prolonged sick leave in subacute low back pain. BMC Musculoskelet Disord. 2009;10(139):2009.

25. Truchon M, Cote D, Schmouth ME, Leblond J, Fillion L, Dionne C. Validation of an adaptation of the stress process model for predicting low back pain related long-term disability outcomes: a cohort study. Spine. 2010;1(35):1307-15.

26. Joy JM, Lowy J, Mansoor JK. Increased pain tolerance as an indicator of return to work in low-back injuries after work hardening. Am J Occup Ther. 2001;55:200-5.

27. Truchon M, Cote D. Predictive validity of the Chronic Pain Coping Inventory in subacute low back pain. Pain. 2005;116:205-12. 
28. Okurowski L, Pransky G, Webster B, Shaw WS, Verma S. Prediction of prolonged work disability in occupational low-back pain based on nurse case management data. J Occup Environ Med. 2003;45:763-70.

29. Van Der Giezen AM, Bouter LM, Nijhuis FJ. Prediction of return-to-work of low back pain patients sicklisted for 3-4 months. Pain. 2000;87:285-94.

30. Hansson TH, Hansson EK. The effects of common medical interventions on pain, back function, and work resumption in patients with chronic low back pain: a prospective 2-year cohort study in six countries. Spine. 2000;1(25):3055-64.

31. Anema JR, Schellart AJ, Cassidy JD, Loisel P, Veerman TJ, van der Beek AJ. Can cross country differences in return-to-work after chronic occupational back pain be explained? An exploratory analysis on disability policies in a six country cohort study. J Occup Rehabil. 2009;19:419-26.

32. Hazard RG, Bendix A, Fenwick JW. Disability exaggeration as a predictor of functional restoration outcomes for patients with chronic low-back pain. Spine. 1991;16:1062-7.

33. Gatchel RJ, Polatin PB, Mayer TG, Garcy PD. Psychopathology and the rehabilitation of patients with chronic low back pain disability. Arch Phys Med Rehabil. 1994;75:666-70.

34. Anema JR, Cuelenaere B, van der Beek AJ, Knol DL, de Vet HC, van Mechelen W. The effectiveness of ergonomic interventions on return-to-work after low back pain; a prospective two year cohort study in six countries on low back pain patients sicklisted for 3-4 months. Occup Environ Med. 2004;61:289-94.

35. Storheim K, Brox JI, Holm I, Bo K. Predictors of return to work in patients sick listed for sub-acute low back pain: a 12-month follow-up study. J Rehabil Med. 2005;37:365-71.

36. Schultz IZ, Crook J, Meloche GR, Berkowitz J, Milner R, Zuberbier OA, et al. Psychosocial factors predictive of occupational low back disability: towards development of a return-towork model. Pain. 2004;107:77-85.

37. Gross DP, Battie MC, Cassidy JD. The prognostic value of functional capacity evaluation in patients with chronic low back pain: part 1: timely return to work. Spine. 2004;15(29):914-9.

38. Gross DP, Battie MC. Predicting timely recovery and recurrence following multidisciplinary rehabilitation in patients with compensated low back pain. Spine. 2005;15(30):235-40.

39. Ilmarinen J. Work ability-a comprehensive concept for occupational health research and prevention. Scand J Work Environ Health. 2009;35:1-5.

40. Vittinghoff E, McCulloch CE. Relaxing the rule of ten events per variable in logistic and Cox regression. Am J Epidemiol. 2007;15(165):710-8.

41. Leeuw M, Goossens ME, Linton SJ, Crombez G, Boersma K, Vlaeyen JW. The fear-avoidance model of musculoskeletal pain: current state of scientific evidence. J Behav Med. 2007;30:77-94.

42. Suissa S. Immortal time bias in pharmaco-epidemiology. Am J Epidemiol. 2008;15(167):492-9.

43. van Walraven C, Davis D, Forster AJ, Wells GA. Time-dependent bias was common in survival analyses published in leading clinical journals. J Clin Epidemiol. 2004;57:672-82.

44. van Kleef E, Green N, Goldenberg SD, Robotham JV, Cookson $\mathrm{B}$, Jit $\mathrm{M}$, et al. Excess length of stay and mortality due to Clostridium difficile infection: a multi-state modelling approach. J Hosp Infect. 2014;18(88):213-7.

45. Frank J, Sinclair S, Hogg-Johnson S, Shannon H, Bombardier C, Beaton D, et al. Preventing disability from work-related low-back pain - New evidence gives new hope - if we can just get all the players onside. Can Med Assoc J. 1998;16(158):1625-31.

46. van Duijn M, Eijkemans MJ, Koes BW, Koopmanschap MA, Burton KA, Burdorf A. The effects of timing on the cost-effectiveness of interventions for workers on sick leave due to low back pain. Occup Environ Med. 2010;67:744-50.
47. De Vet HCW, Heymans MW, Dunn KM, Pope DP, van der Beek AJ, Macfarlane GJ, et al. Episodes of low back pain: a proposal for uniform definitions to be used in research. Spine. 2002;1(27):2409-16.

48. Kongsted A, Kent P, Axen I, Downie AS, Dunn KM. What have we learned from ten years of trajectory research in low back pain? BMC Musculoskelet Disord. 2016;17:220.

49. Steenstra IA, Lee H, de Vroome EM, Busse JW, Hogg-Johnson SJ. Comparing current definitions of return to work: a measurement approach. J Occup Rehabil. 2012;22(3):394-400.

50. Steenstra IA, Koopman FS, Knol DL, Kat E, Bongers PM, de Vet $\mathrm{HC}$, et al. Prognostic factors for duration of sick leave due to lowback pain in dutch health care professionals. J Occup Rehabil. 2005; 15:591-605.

51. Huguet A, Hayden JA, Stinson J, McGrath PJ, Chambers CT, Tougas ME, et al. Judging the quality of evidence in reviews of prognostic factor research: adapting the GRADE framework. Syst Rev. 2013;2:71.

52. Turner JA, Franklin G, Fulton-Kehoe D, Sheppard L, Stover B, $\mathrm{Wu}$ R, et al. ISSLS prize winner: early predictors of chronic work disability: a prospective, population-based study of workers with back injuries. Spine (Phila Pa 1976). 2008;33:2809-18.

53. van der Weide WE, Verbeek JH, Salle HJ, van Dijk FJ. Prognostic factors for chronic disability from acute low-back pain in occupational health care. Scand. 1999;25(25):50-6.

54. Du Bois M, Szpalski M, Donceel P. Patients at risk for long-term sick leave because of low back pain. Spine J. 2009;9:350-9.

55. Heymans MW, De Vet HCW, Knol DL, Bongers PM, Koes BW, van Mechelen W. Workers' beliefs and expectations affect return to work over 12 months. J Occup Rehabil. 2006; $16: 685-95$.

56. Heymans MW, Anema JR, van Buuren S, Knol DL, van Mechelen W, de Vet HC. Return to work in a cohort of low back pain patients: development and validation of a clinical prediction rule. J Occup Rehabil. 2009;19:155-65.

57. Lotters F, Burdorf A. Prognostic factors for duration of sickness absence due to musculoskeletal disorders. Clin $J$ Pain. 2006;22:212-21.

58. Fransen M, Woodward M, Norton R, Coggan C, Dawe M, Sheridan N. Risk factors associated with the transition from acute to chronic occupational back pain. Spine. 2002;27(27):92-8.

59. Baldwin ML, Butler RJ, Johnson WG, Cote P. Self-reported severity measures as predictors of return-to-work outcomes in occupational back pain. J Occup Rehabil. 2007;17:683-700.

60. Nordin M, Skovron ML, Hiebert R, Weiser S, Brisson PM, Campello $\mathrm{M}$, et al. Early predictors of outcome. Bull. 1996;55(55):204-6.

61. Franklin GM, Stover BD, Turner JA, Fulton-Kehoe D, Wickizer TM. Early opioid prescription and subsequent disability among workers with back injuries: the Disability Risk Identification Study Cohort. Spine. 2008;15(33):199-204.

62. Du Bois M, Donceel P. A screening questionnaire to predict no return to work within 3 months for low back pain claimants. Eur Spine J. 2008;17:380-5.

63. Prkachin KM, Schultz IZ, Hughes E. Pain behavior and the development of pain-related disability: the importance of guarding. Clin J Pain. 2007;23:270-7.

64. Goertz MN. Prognostic indicators for acute low-back pain. Spine. 1990;15(15):1307-10.

65. Burdorf A, Naaktgeboren B, Post W. Prognostic factors for musculoskeletal sickness absence and return to work among welders and metal workers. Occup Environ Med. 1998;55(7):490-5.

66. Gross DP, Battie MC, Asante A. Development and validation of a short-form functional capacity evaluation for use in claimants with low back disorders. J Occup Rehabil. 2006;16:53-62. 
67. Gross DP, Battie MC. The prognostic value of functional capacity evaluation in patients with chronic low back pain: part 2: sustained recovery. Spine. 2004;15(29):920-4.

68. Branton EN, Arnold KM, Appelt SR, Hodges MM, Battie MC, Gross DP. A short-form functional capacity evaluation predicts time to recovery but not sustained return-to-work. J Occup Rehabil. 2010;20:387-93.

69. Fulton-Kehoe D, Stover BD, Turner JA, Sheppard L, Gluck JV, Wickizer TM, et al. Development of a brief questionnaire to predict long-term disability. J Occup Environ Med. 2008;50: 1042-52.

70. Institute for Work \& Health. Seven 'principles' for successful return to work. 2007. Institute for Work \& Health. https://www. iwh.on.ca/system/files/documents/seven_principles_rtw_2014_0. pdf.

71. Lambeek LC, van Mechelen W, Knol DL, Loisel P, Anema JR. Randomised controlled trial of integrated care to reduce disability from chronic low back pain in working and private life. BMJ. 2010;16(340):c1035. doi:10.1136/bmj.c1035.:c1035.

72. Hooftman WE, van der Beek AJ, Bongers PM, van Mechelen W. Is there a gender difference in the effect of work-related physical and psychosocial risk factors on musculoskeletal symptoms and related sickness absence? Scand J Work Environ Health. 2009;35:85-95. 